\title{
Effect of heat stress on enzymatic and non-enzymatic antioxidants in Brassica rapa
}

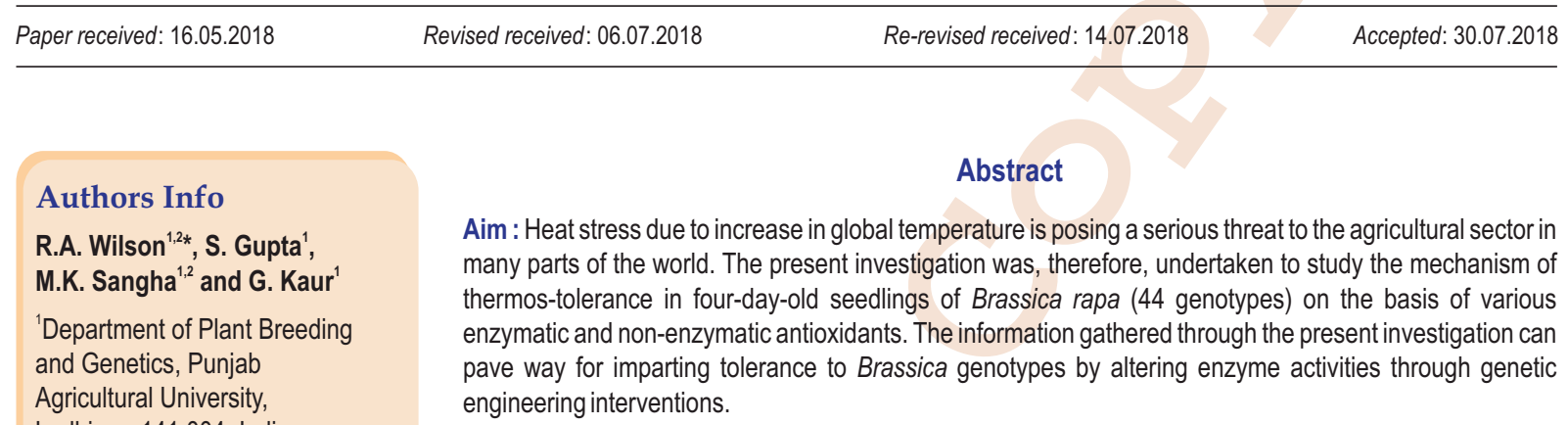

Ludhiana-141 004, India

2Department of Biochemistry,

Punjab Agricultural University,

Ludhiana-141 004, India

Methodology : A total of 44 genotypes were evaluated for survival percentage, electrolyte leakage and chlorophyll content under heat stress conditions. Seedlings were characterized by membrane lipid peroxidation and antioxidants viz. peroxidase and catalase activities, proline and glutathione. Heat stress conditions were created by exposing four-day-old seedlings to $45^{\circ} \mathrm{C}$ for $4.5 \mathrm{hr}$. Out of 44 genotypes, four genotypes (JMT-04-03, TL-2035, TL-98-01 and PBT-37) were thermos-tolerant. Tolerant genotypes registered survival greater than $65 \%$, moderately tolerant between $35-65 \%$ and susceptible less than $35 \%$.

Results : Among various parameters studied, under heat stress, a significant increase in electrolyte leakage, lipid peroxidation, peroxidase activity, glutathione and proline content was observed in comparison to control seedlings, whereas a decline in CAT activity and chlorophyll content was recorded.

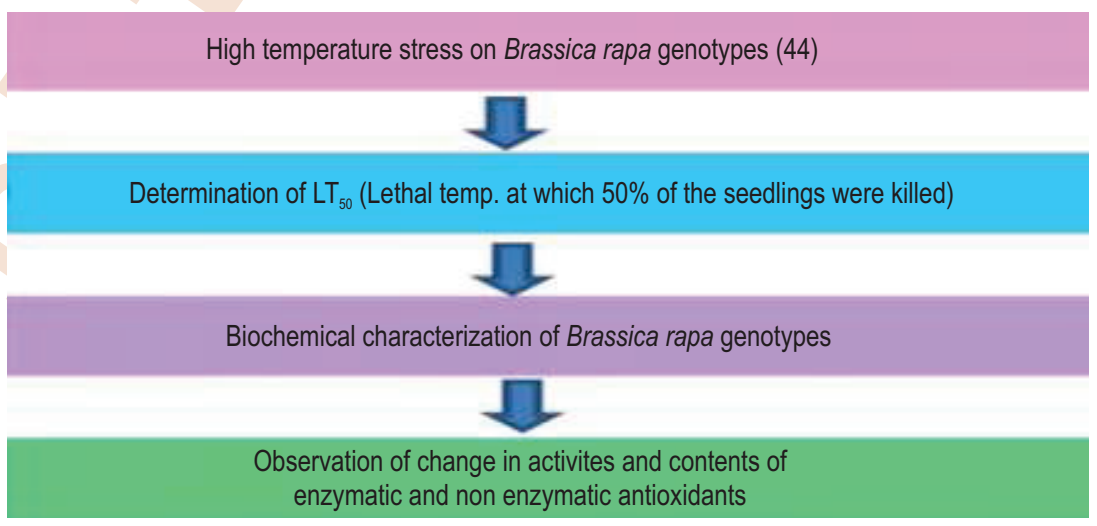

Reviewed by

Dr. Gürcan Güleryüz

Dr. J. K. Datta

Interpretation : Biochemical changes observed in the activities and contents of various parameters studied could be linked with enhanced tolerance to heat stress damage in Brassica rapa which could further be used as a marker for screening against heat stress.

Key words: Antioxidants, Brassica rapa, Heat stress, Lipid Peroxidation, Reactive oxygen species

How to cite: Wilson, R.A., S. Gupta, M.K. Sangha and G. Kaur: Effect of heat stress on enzymatic and non-enzymatic antioxidants in Brassica rapa. J. Environ. Biol., 40, 119-124 (2019). 


\section{Introduction}

Out of various abiotic stresses, high temperature due to global warming is the second most important stress which can strike crop at any time and cause an array of morpho-anatomical, physiological and biochemical changes in plants which impose many limitations on plant growth and development (Kaur et al., 2009; Jukanti et al., 2017). High temperature at sowing time reduces germination, emergence and survival of seedling, resulting in loss of productivity (Wilson et al., 2014). High temperature is further associated with oxidative stress in plants through overproduction of reactive oxygen species such as superoxide radicals $\left(\mathrm{O}_{2}^{-}\right)$, hydroxyl $(\mathrm{OH}-)$, singlet oxygen and hydrogen peroxide $\left(\mathrm{H}_{2} \mathrm{O}_{2}\right)$ (Krishnamurthy and Rathinasabapathi, 2013; Harsh et al., 2016). The overproduced ROS under stress conditions react directly with lipids, proteins and nucleic acids, causing lipid peroxidation mediated membrane injury, protein degradation, enzyme inactivation, pigment bleaching and disruption of DNA structure (Ershova et al., 2011; Sharma et al., 2012). Estimation of lipid peroxidation is a direct index of oxidative stress induced damage due to temperature stress (Pradhan et al., 2013). To counteract the deleterious effects of over-produced ROS, plants have developed scavenging mechanisms categorized as enzymatic and non-enzymatic defence system (Rani et al., 2016).

The species Brassica rapa includes various vegetable and oil crops. Production of these crops is usually impaired by heat stress. $B$. rapa is known to be more sensitive to heat stress as compared to B. juncea and B. napus (Angadi et al., 2000). The optimum temperature required for germination and seed development in Brassica species is $25-33^{\circ} \mathrm{C}$ (Wilson et al., 2014). Previous studies have reported yield loss due to floral sterility and impaired seed filling as the crop experience terminal high temperature stress at grain filling stage $\left(33-35^{\circ} \mathrm{C}\right)$ (Young et al., 2004). Improved thermo-tolerance in plants has been observed due to the synthesis of isoprene or glycinebetaine, production of antioxidant enzymes and reduction in a-linolenic acid concentration (Wahid et al., 2007, Wilson et al., 2014). Survival of the plant during high temperature stress through antioxidant defence system is the urgent need of the time. Research on the effect of high temperature stress on $B$. rapa species is limited in comparison to other Brassica species. Hence, keeping in view the situation leading to crop yield loss, the present research was designed with the objective to understand the effect of high temperature stress in $B$. rapa genotypes and to evaluate the changes in biochemical and antioxidative defence mechanisms.

\section{Materials and Methods}

The seeds of $B$. rapa (44 genotypes) were procured from Oilseeds Section, Department of Plant Breeding and Genetics, Punjab Agricultural University, Ludhiana. Seeds were surface sterilized with $0.1 \% \mathrm{HgCl}_{2}$ solution and then washed under running tap water followed by distilled water. To analyze heat stress tolerance, 20 seeds of each genotype were germinated in a glass jar (500 $\mathrm{ml}$ capacity) in duplicate. The seeds were grown for four days in an incubator under $16 \mathrm{hr}$ light and $8 \mathrm{hr}$ dark period at $25 \pm 2^{\circ} \mathrm{C}$. Out of two jar sets, one set was used as control and the second set was given heat shock treatment at $\mathrm{LT}_{50}$ i.e., $45^{\circ} \mathrm{C}$ for $4.5 \mathrm{hr}$, after 4 days of germination. $\mathrm{LT}_{50}$ is the lethal temperature at which $50 \%$ seedlings of TL-15 (released check variety of $B$. rapa) were killed. Stress tolerance was estimated on the basis of number of seedlings survived $24 \mathrm{hr}$ after heat shock treatment (Datetal.,1998).

Genotypes were subdivided into categories on the basis of persent survival, i.e., tolerant (having survival greater than $65 \%$ ), moderately tolerant (having survival within the range of 35$65 \%$ ) and susceptible (having survival less than $35 \%$ ). Cell membrane stability (electrolyte leakage), chlorophyll content, lipid peroxidation, peroxidase activity, catalase activity, glutathione content and proline content were measured as described in our previous study (Wilson et al., 2014).

Statistical analyses : The experiments were performed in a completely randomised design (CRD). Paired t-test was performed to analyse the effect of heat stress on seedlings (control vs heat stressed). An alpha level of 0.05 was adopted throughout to reduce Type I statistical errors.

\section{Results and Discussion}

A set of forty-four $B$. rapa genotypes were evaluated under heat stress along with the control for thermo-tolerance $\left(45^{\circ} \mathrm{C}\right)$ in terms of survival percentage. On the basis of survival percentage, four genotypes (JMT-04-03, TL-2035, TL-98-01 and PBT-37) were found to be highly tolerant i.e., high rate of survival at $45^{\circ} \mathrm{C}$ for $4.5 \mathrm{hrs}$; twenty two were moderately tolerant and eighteen were susceptible (Fig.1a). Tolerant genotypes had 3.6 fold higher percent survival $(74.41 \%)$ than susceptible genotypes (20.79\%), and survival (\%) was 50.82 i.e., 2.5 fold higher than susceptible genotypes in case of moderately tolerant ones.

Electrolyte leakage data, an indicator of cell membrane stability during heat stress (Wilson et al., 2014), supported the survival data as it increased significantly $(p<0.001)$ under heat stress in seedlings of all the three categories of genotypes as compared to their respective controls (Fig. $1 \mathrm{~b}$ ). In response to heat stress, tolerant genotypes registered $48.26 \%$ of electrolyte leakage, which was two-fold higher than control. In moderately tolerant and susceptible genotypes, it was $58.83 \%$ and $64.50 \%$ which was 2.5 and 3.15 fold higher than their respective controls. The increase in electrolyte leakage during heat stress indicated that heat stress caused cell membrane damage in seedlings, though damage being higher ( $1.5 \mathrm{fold}$ ) in susceptible genotypes than the resistant ones. These results are in confirmation with the previous reports of Al-Jebory (2013) in wheat, and Wilson et al. (2014) in B. juncea.

Chlorophyll content (Fig.1c) decreased significantly $(p<0.05)$ in heat stressed seedlings as compared to control in all the test material, suggesting damage to photosynthetic apparatus (Karim et al., 1997). The decrease in chlorophyll 
content was at par in all the three categories. Similar to the present study, earlier Chaitanya et al. (2001) and Jiang and Huang (2001), reported decline in chlorophyll content under drought, heat or combined stress conditions in mulberry and Turfgrass, respectively. The unstressed seedlings of all the categories showed non-significant variation. In some cases, unchanged chlorophyll level has also been reported during drought stress (Hayat et al., 2009).

Control seedlings did not show any significant variation with respect to lipid peroxidation. Under heat stress, lipid peroxidation increased significantly $(p<0.05)$ in all the categories, except tolerant genotype where increase observed was non-significant. Lipid peroxidation was maximum in susceptible genotypes (10.78 $\mu \mathrm{m} \mathrm{MDA} \mathrm{g}^{-1}$ f.wt.) followed by moderately tolerant genotypes (8.06 $\mu \mathrm{m} \mathrm{MDA} \mathrm{g}^{-1}$ f.wt.) and tolerant genotypes (7.67 $\mu \mathrm{m} \mathrm{MDA} \mathrm{g}^{-1}$ f.wt.) (Fig. 1d). Maximum lipid peroxidation in susceptible genotypes could be due to disturbance in balance between the production and scavenging of ROS (Bowler et al., 1992). The present results are supported by the studies of Jiang and Huang (2001) in turfgrass and Wilson et al. (2014) in B. juncea. High temperature affects membrane linked processes due to alteration in membrane fluidity and permeability (Larkindale and Knight, 2002) which inturn is due to lipid peroxidation. The hydroperoxides, thus, formed decompose into oxy, peroxy radicals including malondialdehyde (Rani et al., 2016).

Higher increase in lipid peroxidation in susceptible genotypes leads to more disruption of membrane integrity (Almeselmani et al., 2006). Low cell membrane integrity is a repercussion of lipid peroxidation of membrane lipids caused by ROS generated during stress. ROS generated during heat stress reacts with unsaturated lipids in membranes and cause lipid peroxidation leading to MDA accumulation (Liu and Huang, 2000). MDA, a product of peroxidation of unsaturated fatty acids in phospholipids, is responsible for cell membrane damage (DaCosta and Huang, 2007).

Since lipid peroxidation was estimated in seedlings, it becomes incumbent to estimate the antioxidant status of the seedlings under heat stress. The different antioxidants studied were: peroxidase (POD), catalase (CAT), proline and glutathione (GSH). A significant $(p<0.01)$ increase in POD activity was registered in all the categories in response to heat stress. The average values for POD action observed was highest in tolerant genotypes (460.0 $\Delta \mathrm{E}$ minute ${ }^{-1} \mathrm{~g}^{-1}$ f.wt. of tissue, followed by moderately tolerant (328.13 $\Delta \mathrm{E}$ minute ${ }^{-1} \mathrm{~g}^{-1}$ f.wt. of tissue) and susceptible ones (302.50 $\Delta \mathrm{E}$ minute $\mathrm{g}^{-1}$ f.wt. of tissue). In comparison to controls, percent increase was $45.45 \%, 40.70 \%$ and $30.95 \%$ in tolerant genotypes, moderately tolerant and susceptible genotypes, respectively (Fig.1e). No significant difference in POD activity was recorded in case of control seedlings. The results presented are in agreement with the previous repotrs of Chakraborty and Tongden (2005) and Rani et al. (2016) on Cicer arietinum and B. juncea, respectively. The increase in POD activity could be associated with chlorophyll degradation during heat stress and was induced probably due to increased levels of peroxide radicals as a result of increased lipid peroxidation (Chakraborty and Tongden, 2005).

Catalase activity decreased significantly $(p<0.001)$ in all the three classes under heat stress. The seedlings of susceptible and moderately tolerant categories showed a higher decrease in CAT activity as compared to tolerant one. The decrease was $55.25 \%$ in susceptible seedlings, $48.68 \%$ in moderately tolerant genotypes and $11.85 \%$ in tolerant genotypes, respectively (Fig.1f). The results obtained in the present investigation are in accordance with those observed by Jiang and Huang (2001), who also reported decrease in CAT activity upon heat stress in turfgrass, respectively. The results are also in consonance with the results of He and Huang (2010) in Kentucky bluegrass. Dat et al. (1998) reported decrease in CAT activity by $9.6 \%$ following heat stress. However, in the present study decrease ranged from 10 to $35 \%$. The CAT activity in unstressed seedlings of all categories showed non-significant difference but activity after heat stress varied significantly among seedlings of three categories. Catalase is a peroxisomal enzyme and helps in scavenging of $\mathrm{H}_{2} \mathrm{O}_{2}$. The decrease in CAT activity could be due to its non-robust nature and sensitivity to heat stress (Fadzillah et al., 1996), and thus would not be playing role in scavenging $\mathrm{H}_{2} \mathrm{O}_{2}$ generated during heat stress (Liu and Huang, 2000). In different studies, catalase activity varied under heat stress suggesting that its activity may vary from species to species (Kaur et al., 2009).

Glutathione content was significantly higher in control seedlings of tolerant genotypes in comparison to other two categories. After heat stress, remarkable significant increase $(p<0.001)$ was observed in GSH content (Fig. $1 \mathrm{~g})$ in seedlings of all the three categories of genotypes. Maximum GSH content was

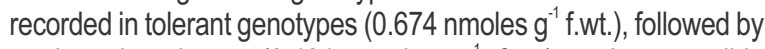
moderately tolerant (0.404 nmoles $\mathrm{g}^{-1}$ f.wt.) and susceptible genotypes (0.293nmoles $g^{-1}$ f.wt.), respectively. Higher GSH content could be responsible for alleviating the oxidative stress due to heat shock as GSH could have detoxified free radicals, and thus act as an antioxidant in heterotrophic as well as in phototrophic tissues (Wise and Naylor, 1987). $\mathrm{H}_{2} \mathrm{O}_{2}$ accumulated during heat stress is quenched by GSH, therefore higher glutathione content could plays a defensive role against heat stress generated oxidative stress (Noctor and Foyer, 1998). The increased GSH levels not only protect against free radicals but also switch the whole panoply of stress resistance response (Noctor and Foyer, 1998; Kumar and chattopadhyay, 2018).

Proline plays a protective role in various stresses (Wahid and Ghazanfer, 2006). In case of control seedlings of all three categories, variation in proline content was non-significant. But heat shock treatment caused significant $(p<0.001)$ increase in proline content, $\sim 1.5$ fold in tolerant and moderately tolerant categories and 1.4 fold in susceptible ones as compared to control. The maximum proline content was found in tolerant genotypes ( $0.136 \mathrm{mg} \mathrm{g}^{-1}$ f.wt tissue), followed by moderately 

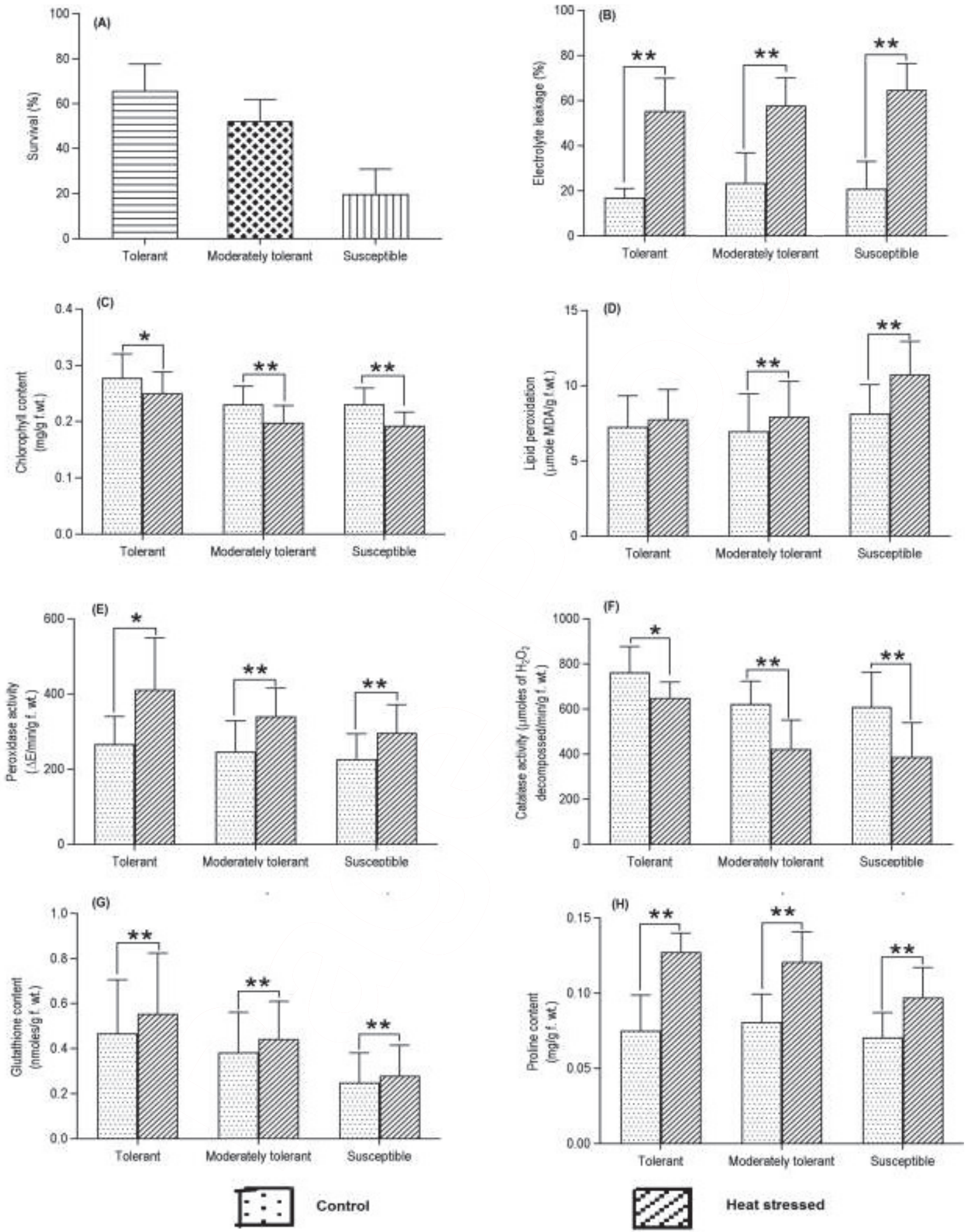

Fig. 1 : Effect of heat stress on (A) percent survival, $(B)$ electrolyte leakage, $(C)$ chlorophyll content, (D) lipid peroxidation, (E) peroxidase activity, $(F)$ catalase activity, $(\mathrm{G})$ glutathione content and $(\mathrm{H})$ proline content of four-day-old $B$. rapa seedlings. Bars and error bars represent mean and standard deviation respectively. Asterisk $\left(^{*}\right)$ and $\left(^{* *}\right)$ brackets represent significant difference between control and heat stressed seedlings at $P<0.05$ and $p<0.01$ level of significance respectively. 
tolerant ( $0.117 \mathrm{mg} \mathrm{g}^{-1}$ f.wt. tissue) and susceptible genotypes (0.100 $\mathrm{mg} \mathrm{g}^{-1}$ f.wt tissue) (Fig.1h). Similar increase in proline content was also reported by Pradhan et al. (2013) in Brassica spp. Proline is an osmolyte that accumulates during stress conditions (Schafleitner et al., 2007) preventing water loss from the cell. (Gupta et al., 2013). Higher proline content in tolerant genotypes is responsible for higher cell membrane stability (Verbruggen and Hermans, 2008). The accumulation of proline, particularly proline residues in protein, provides additional protection against oxidative stress (Ashraf and Foolad, 2007; Harsh et al., 2016) which can be attributed to its cellular redox potential under heat (Wahid et al., 2007) and other environmental stresses like drought and salinity (Ashraf and Foolad, 2007). Increased proline content during heat stress results due to from stimulation of proline synthesis from glutamate (Hayat et al., 2009) by loss of feedback inhibition, decrease in proline oxidation or due to decreased incorporation into proteins (Handa et al., 1986).

The tolerant genotypes with higher survival percentage showed significantly lower $(p<0.01)$ lipid peroxidation than susceptible genotypes, indicating tolerance towards heat induced damage. Biochemically, this heat stress tolerance could be due to higher induction of both enzymatic and non-enzymatic antioxidants (Yildiztugay et al., 2017). The tolerant group showed significantly $(p<0.05)$ higher increase in peroxidase activity in comparison to susceptible group. Likewise, tolerant group showed significantly higher increase in proline $(p<0.05)$ and GSH content $(p<0.01)$ in comparison to susceptible group. The antioxidant defence system has been considered as part of heat stress adaptation and the strength of this system is related to tolerance to heat stress (Wahid et al., 2007; Zandalinas et al., 2017). To conclude, out of forty-four genotypes studied, only four were found to be heat tolerant. The information gathered through present investigation can pave way for imparting tolerance to genotypes via altering enzyme activities through genetic engineering.

\section{Acknowledgments}

The laboratory facilities and infrastructure provided by Oilseeds Section, Department of Plant Breeding and Genetics, Ludhiana and Central Instrumentation Lab, College of Basic Science and Humanities, Punjab Agricultural University is duly acknowledged.

\section{References}

Al-Jebory, E.I.: Changes in cellular membrane tolerance due to heat stress during Triticum sativum L. seeds germination. Mgzn. AlKufa Univ. Biol., 5, 7-15 (2013).

Almeselmani, M., P. S. Deshmukh, R. K. Sairam, S. R. Kushwaha and T. P. Singh: Protective role of antioxidant enzymes under high temperature stress. Plant Sci., 171, 382-388 (2006).

Angadi, S.V., H. W. Cutforth, P. R. Miller, B. G. McConkey, M. H. Entz, S. A. Brandt and K.M. Volkmar: Response of three Brassica species to high temperature stress during reproductive growth. Can. J. Plant Sci., 80, 693-701 (2000).
Ashraf, M. and M. R. Foolad: Roles of glycine betaine and proline in improving plant abiotic stress resistance. Environ. Exp. Bot., 59, 206-216 (2007).

Bowler, C.M., Van Montagu and D. Inze: Superoxide dismutase and stress tolerance. Annu. Rev. Plant Physiol. Plant Mol. Biol., 43, 83-116 (1992).

Chaitanya, K.V., D. Sundar, S. Masilamani and A. R. Reddy: Variation in heat stress-induced antioxidant enzyme activities among three mulberry cultivars. Plant Growth Regul., 36, 175-180 (2001).

Chakraborty, U. and C. Tongden: Evaluation of heat acclimation and salicylic acid treatments as potent inducers of thermo-tolerance in Cicer arietinum L. Curr. Sci., 89, 384-389 (2005).

DaCosta, M. and B. Huang: Changes in antioxidant enzyme activities and lipid peroxidation for bentgrass species in response to drought stress. J. Am. Soc. Hort. Sci., 132, 417-422 (2007).

Dat, J.F., H. Lopez-Delgado, C.H. Foyer and I.M. Scott: Parallel changes in $\mathrm{H}_{2} \mathrm{O}_{2}$ and catalase during thermo-tolerance induced by salicylic acid or heat acclimation in mustard seedlings. Plant Physiol., 116, 1351-1357 (1998).

Ershova, A.N., N.V. Popova and O.S. Berdnikova: Production of reactive oxygen species and antioxidant enzymes of pea and soybean plants under hypoxia and high $\mathrm{CO}_{2}$ concentration in medium. Russ. J. Plant Physiol., 58, 982-990 (2011).

Fadzillah, N.M., V. Gill, R.P. Finch and R.H. Burdon: Chilling, oxidative stress and antioxidant responses in shoot cultures of rice. Planta, 199, 552-556 (1996).

Gupta, N.K., S. Agarwal, V.P. Agarwal, N.S. Nathawat, S. Gupta and G. Singh: Effect of short-term heat stress on growth, physiology and antioxidative defence system in wheat seedlings. Acta Physiol. Plant, 35, 1837-1842 (2013).

Handa, S., A.K. Handa, P.M. Hasegawa and R.A. Bressan: Proline accumulation and the adaptation of cultured plant cells to water stress. Plant Physiol., 80, 938-360 (1986).

Harsh, A., Y.K. Sharma, U. Joshi, S. Rampuria, G. Singh, S. Kumar and R. Sharma: Effect of short-term heat stress on total sugars, proline and some antioxidant enzymes in moth bean (Vigna aconitifolia). AOAS, 61, 57-64 (2016).

Hayat, S., A. Masood, M. Yusuf, Q. Fariduddin and A. Ahmad: Growth of Indian Mustard (Brassica juncea L.) in response to salicylic acid under high-temperature stress. Braz. Soc. Plant Physiol., 21, 187195 (2009).

He, Y. and B. Huang: Differential responses to heat stress in activities and isozymes of four antioxidant enzymes for two cultivars of Kentucky bluegrass contrasting in heat tolerance. J. Am. Soc. Hort. Sci.,135, 116-124 (2010).

Jiang, Y. and B. Huang: Drought and heat stress injury to two cool season turfgrasses in relation to antioxidant metabolism and lipid peroxidation. Crop Sci., 41, 436-442 (2001).

Jukanti, A.K., V.K. Manga, R.K. Bhatt and R. Pathak: Differential response of pearl millet genotypes to high temperature stress at flowering. J. Environ. Biol., 38, 791-797 (2017).

Karim, M.A., Y. Fracheboud and P. Stamp: Heat tolerance of maize with reference of some physiological characteristics. Ann. Bangladesh Agri., 7, 27-33 (1997).

Kaur, P., N. Ghai and M.K. Sangha: Induction of thermo-tolerance through heat acclimation and salicylic acid in Brassica species. Afr. J. Biotech., 8, 619-625 (2009).

Krishnamurthy, A. and B. Rathinasabapathi: Oxidative stress tolerance in plants: Novel interplay between auxin and reactive oxygen species signaling. Plant Signal Behav., 8, 1: e25761 (2013). doi: 10.4161/ psb. 25761

Kumar, D. and S. Chattopadhyay: Glutathione modulates the expression 
of heat shock proteins via the transcription factors BZIP10 and MYB21 in Arabidopsis. J. Exp. Bot., 69, 3729-3743 (2018).

Larkindale, J. and M.R. Knight: Protection against heat stress-induced oxidative damage in Arabidopsis involves calcium, abscisic acid, ethylene and salicylic acid. Plant Physiol., 128, 682-695(2002).

Liu, X. and B. Huang: Heat stress injury in relation to membrane lipid peroxidation in creeping bentgrass. Crop Sci., 40, 503-516 (2000)

Noctor, G. and C.H. Foyer: Ascorbate and glutathione: Keeping active oxygen under control. Ann. Rev. Plant Physiol. Plant Mol. Biol., 49, 249-279 (1998).

Pradhan, S.K, R.C. Gupta and M. Kumar: Effect of 24-Epibrassinolide on lipid peroxidation and proline in three Brassica species under temperature stress. J. Stress Physiol. Biochem., 9, 376-384 (2013).

Rani, B., N. Kumari, Pooja, V. Jain, K. Dhawan, Monika, R. Avtar, A. Kumar and P. Sheoran: Antioxidative system as influenced by high temperature stress in Brassica juncea (L) Czern \& Coss. Curr. Trends Biotechnol. Pharm., 10, 118-125 (2016).

Schafleitner, R., A. Gaudin, R.O.S. Rosales, C.A.L. Aliaga and M. Bonierbale: Proline accumulation and real time PCR expression analysis of genes encoding enzymes of proline metabolism in relation to drought tolerance in Andean potato. Acta Physiol. Plant., 29, 19-26 (2007).

Sharma, P., A.B. Jha, R.S. Dubey and M. Pessarakli: Reactive oxygen species, oxidative damage, and antioxidative defense mechanism in plants under stressful conditions. J. Bot., (2012). doi: 10.1155/ 2012/217037.
Verbruggen, $\mathrm{N}$. and $\mathrm{C}$. Hermans: Proline accumulation in plants: $\mathrm{A}$ review. Amino Acids, 35, 753-759 (2008).

Wahid, A. and A. Ghazanfar: Possible involvement of some secondary metabolites in salt tolerance of sugarcane. J. Plant Physiol.,163, 723-730 (2006).

Wahid, A., S. Gelani, M. Ashraf and M.R. Foolad: Heat tolerance in plant: An overview. Environ. Exp. Bot., 61, 199-223 (2007).

Wilson, R.A., M.K. Sangha, S.S. Banga, A.K. Atwal and G. Shilpa: Heat stress tolerance in relation to oxidative stress and antioxidants in Brassicajuncea. J. Environ. Biol., 35, 383-387 (2014).

Wise, R. and A. W. Naylor: Chilling enhanced photo-oxidation. Evidence for the role of singlet oxygen and superoxide in the breakdown of pigments and endogenous antioxidants. Plant Physiol., 83, 278$282(1987)$

Young, L.W., R.W. Wilen and P.C. Bonham-Smith: High temperature stress of Brassica napus during flowering reduces micro and mega-gametophyte, fertility, induces fruit abortion and disrupts seed production. J. Exp. Bot., 55, 485-495 (2004).

Yildiztugay, E., C. Ozfidan-Konakci, M, Kucukoduk and S.A. Tekis: The impact of selenium application on enzymatic and non-enzymatic antioxidant systems in Zea mays roots treated with combined osmotic and heat stress. Arch Agron Soil Sci., 63, 261-275 (2017).

Zandalinas, S.I., D. Balfagón, V. Arbona and A. Gómez-Cadenas: Modulation of antioxidant defense system is associated with combined drought and heat stress tolerance in citrus. Front Plant Sci., 8, pp. 953 (2017). 